\title{
Finite Element Method for Fluid Structure Interaction with $h p$-Adaptivity
}

\author{
M. A. Abas and R. Abdul-Rahman
}

\begin{abstract}
Recent advances in computational studies of fluid flows with structural interactions suggest that significant contributions have been made towards reliable solutions to the problem. Achieving accurate solutions however remains a considerable task since enormous amount of computer time and memory are usually needed. This applies particularly to a partitioned approach in which structure and fluid are solved separately. This paper attempts to solve fluid-structure interaction (FSI) problems with an $h p$-adaptive finite element method ( $h p$-FEM). The FSI problem is formulated based on a partitioned approach and Arbitrary Lagrangian-Eulerian (ALE) descriptions for the incompressible fluid and structure domains. The $h p$-adaptivity is implemented with an a posteriori error estimator and adaptation to minimize error in energy norm. The automatic mesh adaptation over the triangular mesh is achieved with red-green-blue refinement technique. A strategy for mesh refinement to occur at prescribed key points is used for effective mesh adaptivity. The $h p$-adaptive approach is assessed with traditional uniform mesh refinement and also an $\boldsymbol{h}$-adaptive method on a benchmark test case. From the error convergence, the $h p$-adaptive method is shown to be a viable approach in acquiring accurate solution of a partitioned-based FSI analysis without significant compromise in computational time and memory. It is also found that the convergence of solution in fluid and structure domains is considerably sensitive to the aspect ratios of triangular elements.
\end{abstract}

Index Terms-Fluid structure interaction, finite element analysis, $h p$-FEM, a posteriori error estimates.

\section{INTRODUCTION}

Analysis of fluid flows that are being impeded by an elastic structure poses a particularly challenging task as it is a complex multiphysics problem in which fluid and structure are mutually coupled along a shared boundary. The past few years have seen growing interests in the analysis of fluid-structure interaction (FSI) problems that arise in interdisciplinary fields spanning from biomechanical to microsystems applications [1], [2]. Hence, accurate tools that provide deep understanding of the phenomenon are always of significant interests. Despite considerable advances in the computational analysis of the FSI problems, achieving reasonable accuracy of the solution without excessive computational resources remains an open challenge. This paper attempts to solve the fluid-structure interaction problem with an $h p$-adaptive finite element method $(h p$-FEM), in which a careful decision in local mesh refinement $(h)$ combined with increase in polynomial order

Manuscript received October 5, 2012; revised January 17, 2013. This work was supported in part by the Malaysia Ministry of Higher Education under its fellowships scheme.

The authors are with the School of Mechanical Engineering, Universiti Sains Malaysia, 14300 Nibong Tebal, Penang, Malaysia (e-mail: aizat_abas@yahoo.com; arahman@eng.usm.my). (p) based on an error indicator is expected to result in highly accurate solution. In theory, $h p$-FEM is commonly accepted to yield fastest convergence in a given error norm [3].

To the best of the knowledge of the authors, efforts at using an adaptive FEM approach, particularly $h p$-FEM, in FSI problems are still rather sparse. Substantial early progress in this respect is arguably made by Dunne and Rannacher and Richter and Wick who proposed $h$-adaptive methods with goal-oriented error estimators [4]. Of particular interest is more recent effort by van der Zee et al. The authors have implemented an $h p$-adaptive method for FSI problem which is also based on a goal-oriented error estimator. Their numerical analysis is performed rigorously on a fluid domain with elastic boundary rather than elastic domain [5]. Unlike in the aforementioned works, our approach is however more direct: Firstly, the $h p$-adaptivity is implemented on top of a usual partitioned-based FSI formulation with arbitrary Lagrangian-Eulerian (ALE) description of the fluid and structure domains. It is expected that an iterative solver used is more stable since the resulting equations are smaller and generally better conditioned [6]. The partitioned approach involves separate solutions for fluid and structure domains, together with iterations for each solution to converge to a specified stopping criterion that satisfies both domains as well as the interface conditions. Secondly, the adaptivity is driven to converge in the global errors in energy norm separately for the fluid and structure domains. A posteriori error indicator is used based on a residual estimator of each element. Thirdly, a simple algorithm to decide between the $h$ and $p$-adaptivity is constructed using prescribed key points at certain locations in the domain of the problem following the approach in [7]. Finally, since the domain is to be discretized with triangular elements, a red-green-blue refinement technique is used for mesh adaptivity.

\section{FSI FORMULATION}

In modelling the equations for FSI, the usual Newtonian incompressible fluid governed by the conservation of momentum and mass is used. The structure domain is modelled using St. Venant Kirchoff (STVK) material model [8]. Since structure usually resides in Lagrangian framework while fluid in Eulerian framework, the conventional ALE framework to describe both structure and fluid formulation in one common framework is used [9]. The ALE framework allows the node of the elements to move in normal Lagrangian framework or held fixed in Eulerian manner. It is commonly accepted to combine the best feature of pure Lagrangian framework to track the free surface and pure Eulerian framework to handle large distortion in a mesh. The combined FSI formulations are described by the following 
equations based on the formulations in [9]. Let $\Omega_{f}$ be the fluid domain bounded by the boundary $\partial \Omega_{f}$, then velocity vector of fluid $v$ and scalar field pressure in fluid $p$ are given by:

$$
\begin{aligned}
\left.\left(\left.\rho \partial_{t} v\right|_{x_{0}}+\rho[(v-w) \cdot \nabla v)\right], \psi\right)_{\Omega_{f}}+\left(\nabla \cdot \sigma_{f}, \psi\right)_{\Omega_{f}} \\
=\left(g_{1}, \psi\right)_{\partial \Omega_{f}}+\left(f_{1}, \psi\right)_{\Omega_{f}} \\
(\operatorname{div}(v), \psi)=0
\end{aligned}
$$

where $w$ is mesh velocity, $\rho$ is fluid density, $\partial_{t}$ denotes time derivative, $\psi$ is the basis function, and $g_{1}$ and $f_{1}$ denote the source terms for the boundary and domain, respectively. $\sigma_{f}$ is Cauchy stress tensor of the fluid domain with $v_{f}$ being dynamic viscosity of fluid. Equations (1) and (2) denote the conservation of mass and momentum for fluid cast in ALE description. For the structural problem, letting $\Omega_{s}$ be the structure domain, the deformation may be then expressed with stress tensor $\sigma_{s}$ :

$$
\begin{gathered}
\sigma_{f}=-p I+2 \rho v_{f} \varepsilon_{f}(v), \quad \varepsilon_{f}(v)=\frac{1}{2}\left(\nabla v+\nabla v^{T}\right) \\
\left(\left.\rho \partial_{t} v_{s}\right|_{x_{0}}, \psi\right)_{\Omega_{s}}+\left(\nabla \cdot \sigma_{s}, \psi\right)_{\Omega_{s}} \\
=\left(g_{2}, \psi\right)_{\partial \Omega_{s}}+\left(f_{2}, \psi\right)_{\Omega_{s}}
\end{gathered}
$$

The velocity field is defined as $v_{s}=\partial_{t} u^{s}$ with $u^{s}$ being the displacement vector of the structure. The Cauchy stress tensor, $\sigma_{s}$ is given by

$$
\sigma_{s}=2 \mu \varepsilon_{s}+\lambda\left(\operatorname{tr}\left(\varepsilon_{s}\right)\right) I
$$

where $\mu$ and $\lambda$ are the Lamé constants. The Green-Lagrange strain tensor of structure $\varepsilon_{s}$ is expressed in terms of deformation gradient $F$ as

$$
\varepsilon_{s}=\frac{1}{2}\left(F^{T} F-I\right), F=(I-\nabla u)
$$

where $F$ is the deformation gradient.

At the interface of fluid and structure, the mesh update equation is required for mapping of the computational mesh to the altered geometry. The deformation of the altered geometry as a result of FSI can be expressed as

$$
\left(\nabla \cdot C: \varepsilon\left(u^{s}\right), \psi\right)_{\Omega_{s}}=0
$$

$C:\{\cdot\}$ denotes deformation based on the STVK material model

$$
C: \varepsilon_{s}\left(u^{s}\right)=2 \mu \varepsilon_{s}+\lambda\left(\operatorname{tr}\left(\varepsilon_{s}\right)\right) I
$$

where parameters $\mu, \lambda, \varepsilon_{s}, F$ are as described before.

\section{A POSTERIORI ERROR ESTIMATE}

The a posteriori error estimate is based on locally evaluated residuals. For each element $K$ in $\Omega_{i}$ bounded by either internal edge $\Gamma_{i}$ or boundary edge $\Gamma$, the residual of the element $\eta_{K}$ is given by [10]

$$
\begin{aligned}
\eta_{K}^{2}= & \frac{h_{K}^{2}}{p_{K}^{2}} \int\left|\nabla \cdot q_{h}+f\right|^{2} d \Omega_{i} \\
& +\sum \frac{h_{K}}{2 p_{K}} \int\left|\left[q_{h} \cdot n_{e}\right]_{e}\right|^{2} d \Gamma_{i} \\
& +\sum \frac{h_{e}}{2 p_{e}} \int\left|q_{h} \cdot n_{e}-g\right|^{2} d \Gamma
\end{aligned}
$$

where $q_{h}$ is the computed flux vector in either fluid or structure domain denotes the jump across $e$, with $n_{e}$ being a unit normal to the edge. We denote $h_{K}$ as the size of the element while $h_{e}$ the size of the edge. The last term represents residual with respect to the boundary condition. We note that (4) is applicable to both fluid and structure domains. The first term on the right hand side of (5) measures the local residual of fluid based on Navier-Stokes equation (1) and (2) for fluid domain. For structure domain the residual comes from the Lagrangian STVK formulation of (3). The second term measures discontinuity of the computed flux inside $\Omega$ while the third term is for the boundary $\Gamma$.

The global error indicator $\eta$ is as usual the sum of $\eta_{K}$ over all the elements

$$
\eta^{2}=\sum_{K} \eta_{K}^{2}
$$

\section{HP-ADAPTIVE STRATEGY}

The approach for adaptivity taken in this work follows the one presented in [7]. It is well known that $h$-adaptivity is better suited in a class of problem where the smoothness of solution is compromised. In practice, this occurs when geometric singularities are present the domain. In FSI problems, the fluid domain in the vicinity of fluid-structure interface is assumed to not have sufficiently smooth solution due to the mesh interface so that $h$-adaptivity is preferred. To enforce $h$-adaptivity, "key points" are introduced at predetermined nodes in the mesh. Hence, $h$-adaptivity is prescribed around the key points while $p$-adaptivity anywhere else. The following describes in detail the $h p$-adaptive strategy:

1) Sort the error indicator $\eta_{K}$ in decreasing value.

2) For top $5 \%$ of $\eta_{K}$, check if the element node exist at keypoints

- IF YES

- mark longest edge of element for Green refinement and maintain current $p$

- ELSE

- increase $p$ while leaving the element patch unchanged

3) Find first and second level neighbours of $h$-marked element and compute arc angle of the neighbours that lie opposite to the marked edge.

- IF arc angle < 50。,

- Mark all edges for Red refinement

- ELSE IF arc angle $>50$

- Mark two out of three edges for Blue refinement 


\section{Test CASE: Elastic Driven CAVity}

The effectiveness of the $h p$-adaptive implementation is investigated on a simple stationary test case problem, namely the modified version of the classical lid driven cavity problem as used in [4]. Fig. 1 shows the domain described as follows:

$$
\Omega=(0,1)^{2} \subset R^{2}
$$

is split into the lower solid part

$$
\Omega_{s}=(0,1) \times(0,1 / 4)
$$

and the upper fluid part

$$
\Omega_{f}=(0,1) \times(1 / 4,1) .
$$

A smooth Dirichlet inflow $x$-directional velocity is prescribed at the top part of the boundary with $v_{x}=4 x(1-x)$. For ease of computation, all other boundaries of $\Omega$ are given homogenous Dirichlet boundary conditions for velocity. Incompressible fluid with density $\rho_{f}=1 \mathrm{kgm}^{-1}$ and dynamic viscosity $v_{s}=0.01 \mathrm{kgm}^{-1} \mathrm{~s}^{-1}$ is prescribed. The structure part is of density $\rho_{s}=1 \mathrm{kgm}^{-1}$, Poisson ratio 0.3 and Lamé parameter $\mu=0.05$.

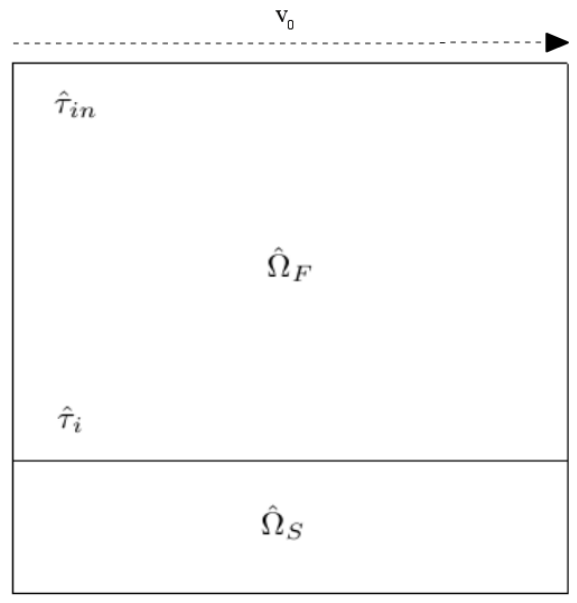

Fig. 1. Elastic driven cavity test case.

\section{DISCUSSIONS}

Fig. 2 demonstrates first and second levels of $h p$-adaptivity. In the second adaptive level, mesh refinement occured at the FSI interface where the key points are prescribed whereas other elements further from them experienced increased in polynomial orders. Beyond the second adaptivity level, no elements had the nodes coinciding with key points and having largest error. Thus strictly $p$-adaptation took place for elements with highest errors. To see the effect of key points, an extra set of key points was added in the middle of FSI mesh. These extra key points were prescribed given the fact that elements with highest error indicator were present near the fluid-structure interface and the top boundary as depicted in Fig. 2(a). To compare the efficiency of $h p$-adaptive keypoint strategy, Fig. 3 shows the plots of the normalized global error against number of DOF for different adaptive methods.

As expected, considerably faster convergence rate is observed for $h p$-adaptive strategy on the fluid domain compared to other methods as seen in Fig. 3(a). However, the $h$-adaptive method has reached the lowest estimated error suggesting better stability of the iterative solver and preconditioner used, namely Biconjugate Gradient-Stabilized and Incomplete LU (BiCGS-ILU). The $h p$-adaptive strategy however shows a better potential to achieve the lowest error with smallest number of DOF if the stability of the solver can be improved.

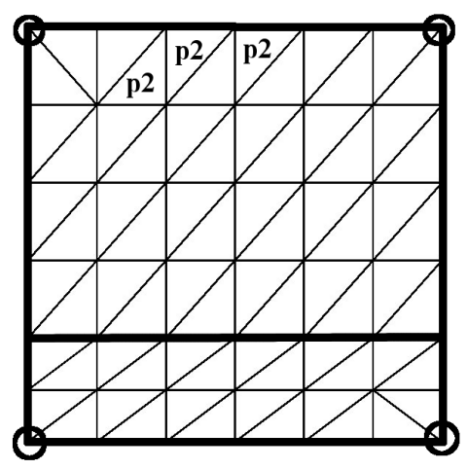

(a) First level $h p$-adaption. Higher order elements $(p=2)$ are correctly introduced in the region of high velocity.

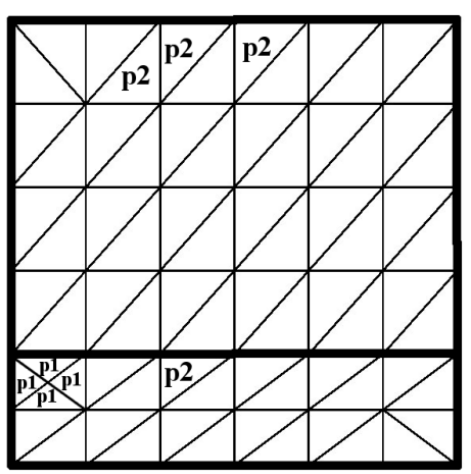

(b) Second level $h p$-adaption. Elements near FSI interface at the keypoint are refined as expected.

Fig. 2. First two levels of hp-adaption. Second order elements are labeled as " $p 2$ "; the rest are first order elements.

For the structure domain, the potential for the $h p$-adaptive strategy to yield substantial improvement is even higher as seen in Fig. 3(b). Error is reduced swiftly within first and second level $h p$-refinement although the stability of the solver seemed to be affected for the subsequent levels, resulting in deteriorating convergence. On the other hand, the other methods apparently are more unstable as suggested by the lack in convergence. Even though the $h p$-adaptive key point strategy is shown as the most promising, its effectiveness is in some cases dependent on the choice of prescribed key points. Increasing the number of key points especially at the region of fluid structure interface or at the point of load applications is deemed to enhance the convergence rate even further. Fig. 4 shows the improvement in mesh refinement with three additional keypoints. In terms of elements distortion, the $h p$-adaptive method as depicted in Fig. 4 managed to maintain all triangular elements within acceptable aspect ratios which in turn assisted both structure and fluid solver for better convergence and stability of numerical computations as previously shown in Fig. 3. 


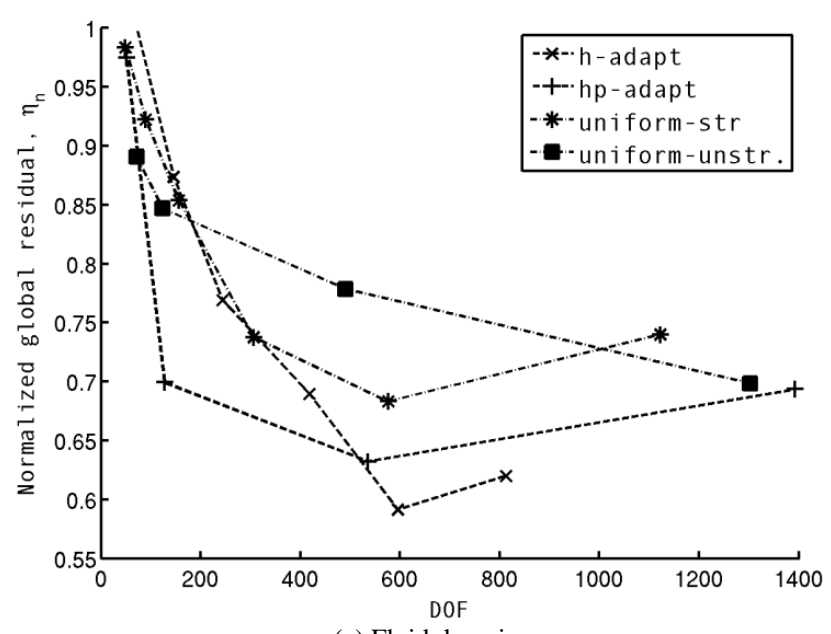

(a) Fluid domain.

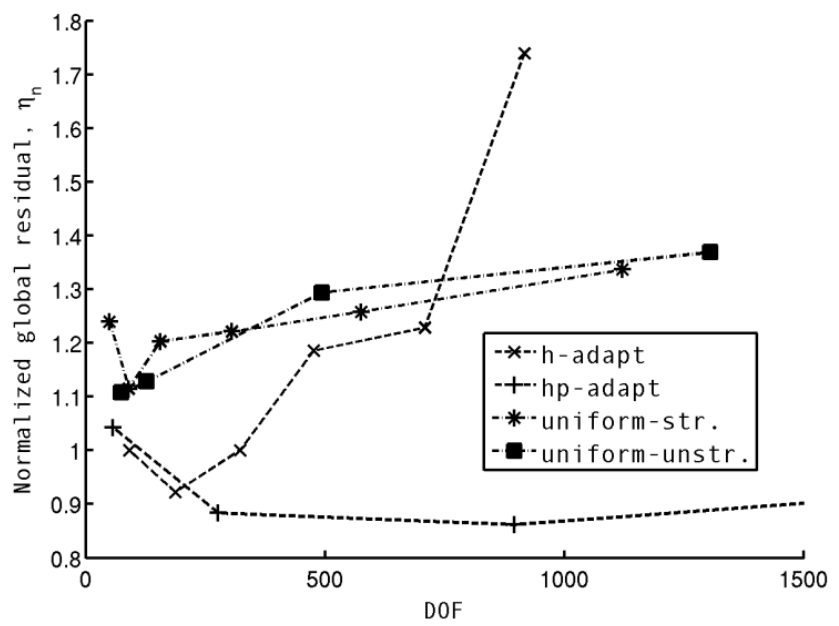

(b) Structure domain.

Fig. 3. Comparison of the convergence rates for structure and fluid domains between $h-, h p$-adaptive and uniform refinements (structured and unstructured).

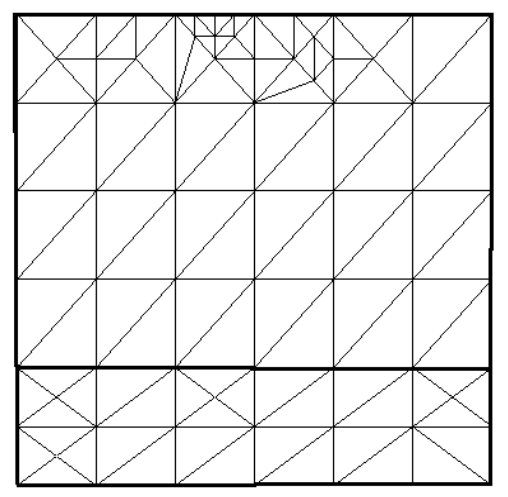

Fig. 4. Sixth level $h p$-adaptive with extra key points denoted with the circles. Three extra key points are added in the middle of the region.

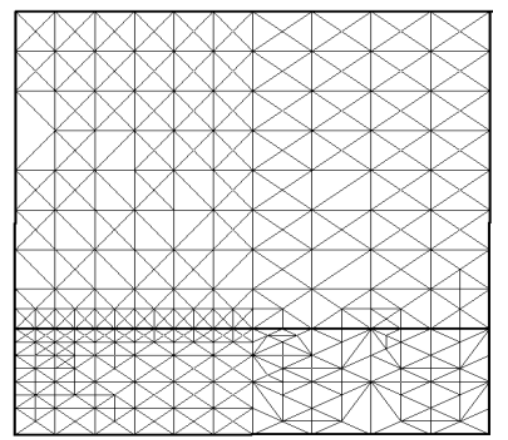

Fig. 5. Sixth level $h$-adaptive with some indication of mesh deterioration in the structure domain.

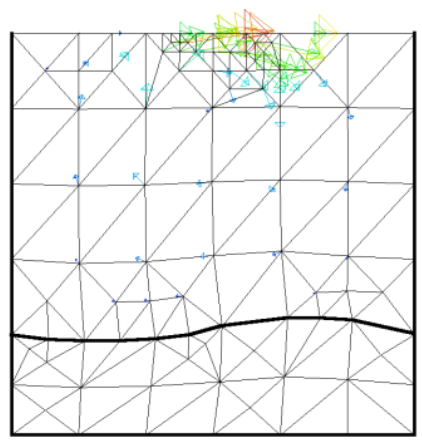

Fig. 6. Absolute velocity vector distributions across fluid domain depicting relatively high absolute velocity at the top the fluid domain.

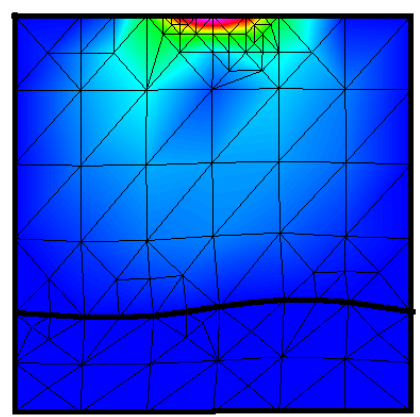

Fig. 7. Absolute velocity distribution across cavity after $12 \mathrm{hp}$-adaptive levels.

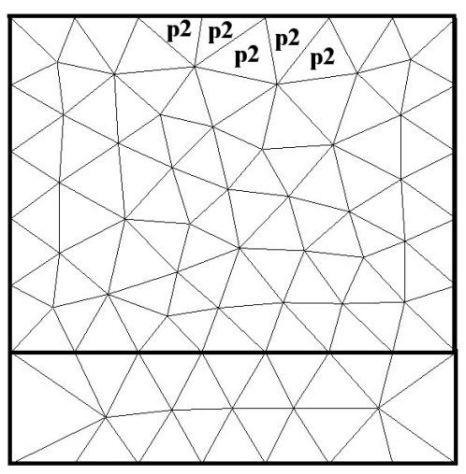

(a) First level $h p$-adaption showing second order elements (p2). The rest are linear elements.

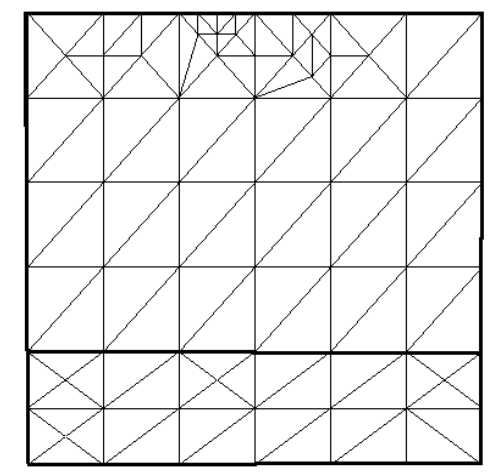

(b) Third level hp-adapt with extra keypoints.

Fig. 8. $h p$-adaption with unstructured mesh.

In contrast, increasing number of element as a result of $h$-refinement of element means higher probability of triangular elements to distort. Fig. 5 shows the occurence of a few highly distorted elements at the vicinity of fluid structure interface. Fig. 6 illustrates the velocity vector distribution while Fig. 7 illustrates its corresponding magnitudes for the cavity FSI problem. Of significance is both figures show the displacement distributions at the interface of FSI as a result of 
information transfer between fluid and structure. Fig. 8 depicts the $h p$-adaptive method on an unstructured mesh. Apparently, increasing the number of key points as introduced in Fig. 4 did not improve the convergence rate of error compared to the structured mesh. Nevertheless, unstructured mesh could reach lower estimated residual error compared to structured mesh in the fluid domain. This could be due to the applied horizontal velocity at the top boundary that resulted in rotating motion of the fluid which was better captured by unstructured mesh. In terms of degeneration of elements, the unstructured mesh was found to have less degenerated elements and more elements with better aspect ratios compared to structured mesh. This attribute makes it better suited for application on more geometrically complex domains. Comparing the structured and unstructured triangular meshes, it is quite apparent that the adaptive method for the FSI problem is rather highly sensitive to the quality of the mesh.

\section{CONCLUSIONS}

This paper has demonstrated a potential approach for an efficient and cost-effective $h p$-adaptive method for solving partitioned-based FSI problems. The $h p$-adaptive method has been shown to yield good performance in terms of its convergence rate compared to other adaptive method. Furthermore, the $h p$-adaptive method achieves its lowest estimated relative error with the smallest number of DOFs which suggests considerable saving in computation time. Despite increasing mesh number near key points, some efforts have been made to retain all the triangular elements within an acceptable aspect ratio. This contributed to a better convergence especially when solving with the partitioned approach that seems to be highly sensitive to mesh distortion. The $h p$-adaptive key point strategy shows effectiveness in handling solution in the vicinity of the fluid-structure interface. In comparison of both cases of uniform refinement, unstructured mesh shows a better convergence rate than structured mesh.

\section{REFERENCES}

[1] A. Quaini and A. Quarteroni. (2009). Algorithms for fluid-structure interaction problems arising in hemodynamics. EPFL, Lausanne. [Online]. Available: http:// http://infoscience.epfl.ch/record/129770?
[2] Y.-Y. Tsui and S.-L. Lu, "Evaluation of the performance of a valveless micropump by CFD and lumped-system analyses," Sensors and Actuators a Physical, vol. 148, pp. 138-148, 2008.

[3] O. C. Zienkiewicz and R. L. Taylor, The Finite Element Method, the Basis, 5th ed. Oxford, UK: Elsevier, vol. 1, pp. 401-426, 2000.

[4] T. Dunne and R. Rannacher, "Adaptive finite element simulation of fluid structure interaction based on an eulerian variational formulation," in Fluid-Structure Interaction: Modelling, Simulation, Optimization, H.-J. Bungartz and Michael Schäfer, Eds. Berlin: Springer Verlag, 2006, pp. 1-75.

[5] K. G. V. D. Zee, E. H. V. Brummelen, I. Akkerman, and R. D. Borst, "Goal-oriented error estimation and adaptivity for fluid-structure interaction using exact linearized adjoints," Computer Methods in Applied Mechanics and Engineering, vol. 200, no. 37, pp. 2738-2757, 2011.

[6] P. Ryzhakov, R. Rossi, S. Idelsohn, and E. Onate, "A monolithic Lagrangian approach for fluid-structure interaction problems," Computational Mechanics, vol. 46, pp. 883-899, 2010.

[7] M. Schober and M. Kasper, "Comparison of hp-adaptive methods in finite element electromagnetic wave propagation," The International Journal for Computation and Mathematics in Electrical and Electronic Engineering, vol. 26, no. 2, pp. 431-446, 2007.

[8] T. Richter and T. Wick, "Finite elements for fluid-structure interaction in ALE and Fully Eulerian coordinates," Computer Methods in Applied Mechanics and Engineering, vol. 199, no. 41-44, pp. 2633-2642, 2010.

[9] J. Donea, A. Huerta, J. P. Ponthot, and A. R. Ferran, Arbitrary Lagrangian Eulerian Methods. Oxford, UK: Wiley, 2004.

[10] J. M. Melenk and B. I. Wohlmuth, "On residual-based a posteriori error estimation in hp-FEM," Advances in Computational Mathematics, vol. 15, pp. 311-331, 2011.

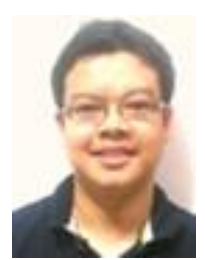

Mohamad Aizat Abas was born in Penang, Malaysia on the $26^{\text {th }}$ of July 1985 . He obtained Bachelor (BEng) and Master (MEng) degrees in Mechanical Engineering at the University of Nottingham, Nottingham, United Kingdom in 2008 and 2009, respectively. $\mathrm{He}$ is currently pursuing the Doctor of Philosophy degree a the School of Mechanical Engineering, Universiti Sains Malaysia. His current field of interest is related to adaptive methods in fluid-structure interaction systems.

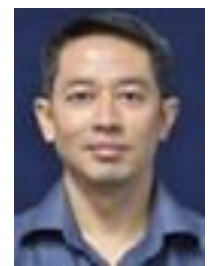

Razi Abdul-Rahman was born in Penang, Malaysia. $\mathrm{He}$ received the B.S. degree in Mechanical Engineering from Carnegie Mellon University, Pittsburgh, PA, in 1996, and the M. S. degree in Mechatronics Engineering and the Ph.D. degree in engineering from Hamburg University of Technology, Hamburg, Germany in 2002 and 2008, respectively. A lecturer at the School of Mechanical Engineering, Universiti Sains Malaysia, his current research interests include adaptive finite element methods for multiphysics systems. 